\title{
Electrosensory Response Mechanisms in Mormyrid Electric Fish ${ }^{1}$
}

\author{
Patrick D. Roberts \\ Neurological Sciences Institute, OHSU 1120 N.W. 20 th Ave. Portland, OR 97209, \\ E-mail: proberts@reed.edu
}

\begin{abstract}
Mormyrid electric fish use mormyromast receptors in their skin to detect distortions in a self-generated electric field. The electroreceptor afferents modulate neuronal activity in the electrosensory lateral line lobe (ELL), a cerebellum-like structure. In this study of the ELL's mormyromast region, computer simulations compare mechanisms responsible for the observed responses involved in active electrolocation with the ampullary region's responses involved in sensing fields of external origin. The simulations are based on a mathematical characterization of the relevant anatomy, physiology, and rules of synaptic plasticity that influence neuronal activity in the ELL, as determined by experimental studies.
\end{abstract}

Key words: Electrosensory; Learning; Synaptic plasticity

\section{Introduction}

The purpose of this study is to resolve an apparent paradox in our understanding of the initial processing of electrosensory information in mormyrid electric fish. Adaptation to predictable reafferent input increases sensitivity to novel stimuli [8], but reduces sensitivity required for electrolocation. The fish resolves this paradox by devoting distinct electroreceptors to each function. The primary afferents of these two of receptor types terminate in different regions of the electrosensory lateral line lobe (ELL). This study uses modeling to investigate the differences in physiology between these two regions of the ELL yielding an explanation of the differences in function. The simulations

1 The author would like to thank Curt Bell for an exciting collaboration and Gin McCollum for discussions and helpful suggestions on the manuscript. This research is supported in part by the National Science Foundation grant IBN-9808887. 
described here investigate mechanisms that allow the neurons in the electrolocation pathway to maintain their sensitivity to electrosensory reafferent information while adapting to predictable changes in those signals.

The mormyrid electric fish uses a specialized electric discharge organ to generate electric pulses. The fish can detect electric fields in its environment by using electrosensory receptors in its skin. This electrosensory system is employed by the fish for the tasks of active electrolocation, communication, and detection of external electrical sources [2].

Two types of electroreceptors are important for the present study. Ampullary receptors are tonically active with an output frequency that is modulated by electric field intensity across the surface of the skin. These receptors are specialized to detect externally generated low frequency electric signals that alert the fish to predators and prey.

Mormyromast receptors are quiescent without stimulation and respond to rapid changes in the electric fields across the skin with a sharp burst. These receptors are very sensitive to the fish's electric organ discharge and code the intensity of the signal into the latency of their response. It is through this pathway that the information needed for active electrolocation is processed. Subtle distortions of the fish's own electric discharge caused by external objects in the environment are encoded in latencies of the mormyromast afferent spikes.

Electrosensory afferents project to the ELL, a laminar cerebellum-like structure in the mormyrid hindbrain that functions as an adaptive filter to eliminate predictable electrosensory signals. The ELL contains Purkinje-like medium ganglion (MG) cells that exhibit adaptive responses to changes in intensity and timing of the reafferent electric field [3].

When a motor command initiates an electric organ discharge, a signal that is linked to the motor command (corollary discharge) is sent to the ELL. This provides the information necessary to eliminate the predictable reafferent response through a process involving synaptic plasticity $[1,5]$. Synapses that contact MG cells adapt through a process that is very sensitive to the timing of pre- and postsynaptic activity. The temporally dependent learning rule of synaptic change is responsible for sculpting an image of the expected sensory image that is subtracted from the afferent input. Previous modeling studies of the ampullary pathway [7] have revealed that the rules of synaptic change, in combination with a series of adaptable synaptic inputs linked to the corollary discharge, result in the cancellation of predicted sensory inputs.

However, the results of reafferent image cancellation are not observed in the mormyromast region. In the mormyromast region, the activity of medium ganglion cells reflects the burst pattern of the mormyromast afferents, a response 


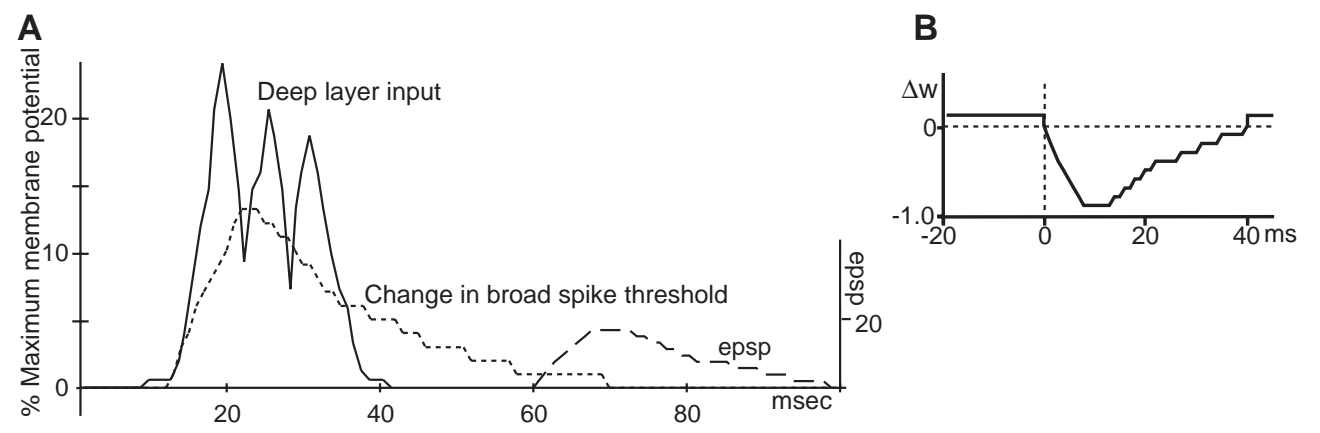

Fig. 1. Functional waveforms in the model. (A) Non-adaptive deep layer in put with three peaks (solid) in units of percent of maximum average membrane potential. Waveform for parallel fiber epsps (broken) in arbitrary units. The time dependent change of the broad spike threshold (dotted) in the final simulation, Fig. 2D. (B) Timing relation for parallel fiber synaptic learning rule. Depression occurs if a broad spike follows the beginning of an epsp by less than 40 msec, otherwise the weight $(w)$ of the synapse is enhanced.

that does not appear to be cancelled by adaptive processes.

The present study investigates the possible mechanisms responsible for the observed MG cell responses in the mormyromast region. We begin with the most obvious differences from the ampullary region, the strong corollary discharge input to deep layers of the mormyromast region, and then modify the model with physiologically realistic assumptions that explain the differences in neuronal responses.

\section{The Model}

We model the MG cell as a single-compartment stochastic neuron with membrane potential that is the sum of synaptic inputs and noise [6]. The model includes two classes of inputs: parallel fiber and deep layer inputs.

Parallel fibers in the molecular layer of the ELL evoke excitatory postsynaptic potentials (epsps). Parallel fibers carry corollary discharge signals linked to the electric organ discharge motor command. These corollary discharge signals are delayed so that a series of epsps arrive up to $100 \mathrm{msec}$ following the electric organ discharge [4]. The total contribution of the parallel fibers in the model neuron is the weighted sum of epsp waveforms. The epsp waveform used in the simulation is taken from in vitro measurements (Fig. 1A).

The deep layer input is the sum of electrosensory afferent information and a corollary discharge signal in the form of a brief burst at the beginning of every electric organ discharge cycle. This is represented in the model by a non-adaptive input derived from in vivo experiments (Fig. 1A). 
The sum of parallel fiber and deep layer inputs yields the average membrane potential. This average potential is added to Gaussian noise to yield the probability that the combination is above threshold for two different kinds spikes. One spike with a lower threshold, the narrow (axonal) spike, is the carrier of information to the next cell. A second spike with a higher threshold, the broad (dendritic) spike, propagates into the apical dendrites and is necessary for associative depression of the parallel fiber synapses. In the simulation, a random number generator determines whether spikes are assigned at each (1 msec) time step using a probability function computed with a sigmoid function of the average membrane potential (see [7] for details).

In the simulation, synaptic weights of parallel fiber inputs are adjusted each cycle by the learning rule measured in vitro [5] and shown in Fig. 1B. If a broad spike occurs during the epsp of a particular synapse, then the weight of that synapse is depressed proportionally to the size of the epsp at each time step. Otherwise, the weight is increased by the predetermined non-associative learning rate (Fig. 1B).

\section{$3 \quad$ Results}

Three simulations were carried out. The first showed that when the model parameters used in previous studies of the ampullary region are applied to the mormyromast region, the model results disagree with known experimental results. The next two simulations add biologically realistic assumptions yielding an improved match between the model andexperiment.

The first simulation was run with a series of corollary discharge signals and noise as in previously successful simulations of the ampullary region. This simulation of the mormyromast region differed in that the deep layer input was based on the mormyromast receptor afferents plus a corollary discharge input. As can be seen in the spike histogram in Fig. 2B, the system attempts to cancel the deep layer input through synaptic change in the molecular layer rendering a rather flat, tonic output. Although a burst is still visible, the model neuron is active throughout the cycle. This result differs from the MG cells of the mormyromast region observed in experiments in vivo shown in Fig. 2A.

It is possible to reduce the tonic narrow spike activity of the model neuron following the burst by reducing the level of noise. Further improvements result by reducing the difference between the narrow and broad spike thresholds. However, the level of activity cannot be reduced to that observed in vivo without setting the broad spike threshold below that of the narrow spikes. The result of reducing noise along with an increasing variation of the epsp timing is shown in Fig. 2C, which now takes on the characteristic form of 


\section{A}

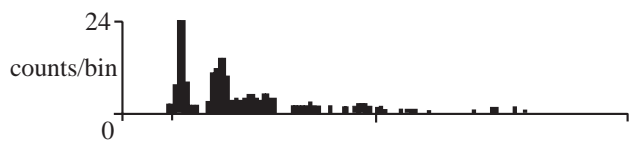

\section{B}

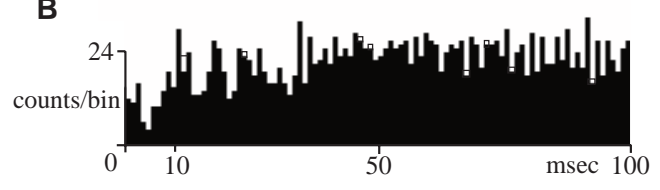

C

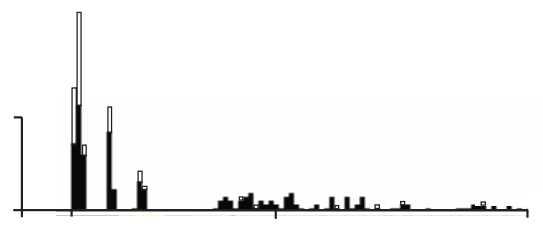

D

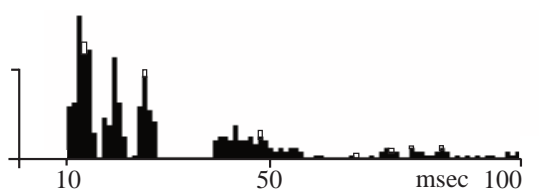

Fig. 2. Comparison of simulation to experimental data. (A) Mormyromast MG neuron activity histogram for first $100 \mathrm{msec}$ following electric organ discharge (histogram with 1 msec bins, 75 sweeps) (modified from Bell and Grant, 1992). (B) Simulated data using mormyromast deep layer inputs with parameters that were appropriate for the ampullary region. Filled (open) bars represent narrow (broad) spike counts. Narrow (broad) spike threshold, $45 \%$ (70\%) maximum membrane potential; standard deviation of the noise, $50 \%$. (C) Simulated data with a linearly increasing variation, from 0 to 6 msec, of parallel fiber epsp onset. Narrow (broad) spike threshold, $38 \%$ ( $40 \%$ ); standard deviation of the noise, $20 \%$. (D) Same as (C) with time dependent increase in broad spike threshold given in Fig. 1A.

bursts in the mormyromast region.

There is still disagreement between results of the simulation and experiments conducted in vivo. Although the experimental data displayed in Fig. 2A does not explicitly show the broad spike rates, broad spikes are relatively rare during the burst (C. Bell, private communication). Without further modification of the model, the broad spikes are found to be prominent in the simulation result shown in Fig. 2C. The reduction of broad spike activity, while narrow spike activity remains strong, would imply an increase in the broad spike threshold even though the narrow spike threshold remains constant. The result of such a time dependent change in broad spike threshold is shown in Fig. 2D where the burst is composed overwhelmingly of narrow spikes, in good agreement with experiment.

\section{Conclusion}

This model predicts that the threshold of the broad spikes is increased during the period when the mormyromast and corollary discharge input arrives to the deep layers. In addition, there appears to be less noise in the mormyromast region than the ampullary. These two mechanisms combine to give the MG cells of the mormyromast region a characteristic burst to help define the reafferent information [8] for accurate electrolocation. The simulations presented here 
resolve the apparent paradox that the MG cells in the mormyromast region respond to electric organ discharge in bursts when they ought to adjust their parallel fiber input to cancel the reafferent signal as do the MG cells of the ampullary region.

\section{References}

[1] C C Bell, Properties of a modifiable efference copy in an electric fish, $J$. Neurophysiol. 47 (1982) 1043-1056,

[2] C C Bell, Electroreception in mormyrid fish: Central physiology, In T. H. Bullock and W. Heiligenberg, eds., Electroreception (Wiley, New York, 1986) 423-452 .

[3] C C Bell, A Caputi, and K Grant, Physiology and plasticity of morphologically identified cells in the mormyrid electrosensory lobe, J. Neurosci. 17 (1997) 64096423 .

[4] C C Bell, K Grant, and J Serrier, Sensory processing and corollary discharge effects in the mormyromast regions of the mormyrid electrosensory lobe: I. Field potentials and cellular activity in associated structures, J. Neurophysiol. 68 (1992) 843-858.

[5] C C Bell, V Han, Y Sugawara, and K Grant, Synaptic plasticity in a cerebellumlike structure depends on temporal order, Nature 387 (1997) 278-281.

[6] W Gerstner and J L van Hemmen, Associative memory in a network of 'spiking' neurons, Network 3 (1992) 139-164.

[7] P D Roberts and C C Bell, Computational consequences of temporally asymmetric learning rules: II. sensory image cancellation, J. Compu. Neurosci. (in press, 1999).

[8] E von Holst and H Mittelstaedt, Das Reafferenzprinzip, Naturwiss. 37 (1950) $464-476$.

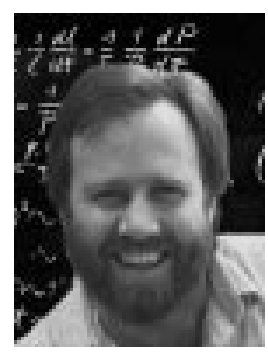

Patrick D. Roberts is a Research Assistant Scientist at the Neurological Sciences Institute (Oregon Health Sciences University) in Portland, Oregon. He completed his undergraduate studies in physics at Reed College in Portland (1983). He received his Ph.D. from the University of Gothenburg, Sweden (1993) in theoretical elementary particle physics where his dissertation research concentrated on theoretical aspects of string theory and conformal field theory. Since completing his graduate degree, Dr. Roberts has been developing mathematical frameworks for applications to neurophysiological systems. These projects include studies of human posture and movement, rhythm generation in small networks, and dynamics of neural activity in the cerebellum and cerebellum-like structures. 